\title{
Pinnae fistula or cyst
}

INSERM

\section{Source}

INSERM. (1999). Orphanet: an online rare disease and orphan drug data base. Pinnae

fistula or cyst. ORPHA:155838

Pinnae fistula or cyst is a rare otorhinolaryngological malformation characterized by the presence of a, usually unilateral, sinus tract or cyst located in the vicinity of the auricle (most frequently identified by a small pit near the anterior marg in of the first ascending portion of the helix). Typically, patients are asymptomatic and usually only present symptoms (pain, erythema, discharge from pit) in relation to infection. Renal and inner ear anomalies may be associated. 